PROCEEDINGS OF THE

AMERICAN MATHEMATICAL SOCIETY

Volume 130, Number 9, Pages 2497-2502

S 0002-9939(02)06609-1

Article electronically published on April 17, 2002

\title{
ON THE NUMBER OF FOURIER COEFFICIENTS THAT DETERMINE A HILBERT MODULAR FORM
}

\author{
SRINATH BABA, KALYAN CHAKRABORTY, AND YIANNIS N. PETRIDIS
}

(Communicated by Dennis A. Hejhal)

\begin{abstract}
We estimate the number of Fourier coefficients that determine a Hilbert modular cusp form of arbitrary weight and level. The method is spectral (Rayleigh quotient) and avoids the use of the maximum principle.
\end{abstract}

\section{INTRODUCTION}

Jacquet, Piatetski-Shapiro and Shalika [4] show that if two cusp forms on $\mathrm{GL}_{n}$ which are Hecke eigenforms have all but finitely many Fourier coefficients equal, then the forms are identically equal. Their argument uses the properties of RankinSelberg $L$-functions. Moreno, using the $L$-series attached to the modular forms, showed that a suitably chosen finite set of Fourier coefficients determines the form uniquely (see [5]). Goldfeld and Hoffstein, in 2], prove a similar result for holomorphic Hecke eigenforms for $\Gamma_{0}(N)$; their bound on the number of initial Fourier coefficients that determine the modular form is $O\left((\log N)^{2}(\log \log N)^{4}\right)$ assuming the Riemann hypothesis for Rankin-Selberg $L$-functions, and $O(N \log N)$ unconditionally.

In 6] Murty improves the results of Goldfeld and Hoffstein by removing the condition that the forms are Hecke eigenforms. Murty shows that a holomorphic cusp form of weight $k$ for $\Gamma_{0}(N)$ is determined by the first $(k / 12) N \prod_{p \mid N}(1+(1 / p))$ Fourier coefficients. This improves the earlier results on the dependence on the level, and gives a linear dependence on the weight of the cusp form. He also improves the bound for Hecke eigenforms to $O\left((\log N)^{2}\right)$.

In [3. Huntley concentrates on non-holomorphic Maaß cusp forms $F$ of weight 0 for cofinite groups acting on products of hyperbolic spaces $\mathbb{H}^{n_{1}} \times \cdots \times \mathbb{H}^{n_{p}}$, where $\mathbb{H}^{n_{i}}$ is the $n_{i}$-dimensional hyperbolic space. At the $j$-th cusp, one has a Fourier expansion with Fourier coefficients $a_{\vec{l}}^{(j)}$, where $\vec{l}$ runs through a lattice $\Lambda$. Using the Rayleigh quotient for the Laplace operator, he shows that if $a_{\vec{l}}^{(j)}=0$ for every $j$ and for all $\vec{l}$ with $|\vec{l}| \leq C|\vec{\lambda}|^{1 / 2}$, where $\vec{\lambda}=\left(\lambda_{1}, \ldots, \lambda_{p}\right), \lambda_{i}$ is the eigenvalue of the Laplacian acting on $\mathbb{H}^{n_{i}}$, and $|\vec{l}|$ denotes the length of the vector $\vec{l}$, then $F$ is identically zero. He proceeds to show that the spectral multiplicity $m(\vec{\lambda})$ of $\vec{\lambda}$ satisfies a bound $m(\vec{\lambda})=O\left(|\vec{\lambda}|^{(n-p) / 2}\right)$, where $n=\sum_{i} n_{i}$.

Received by the editors February 27, 2001.

2000 Mathematics Subject Classification. Primary 11F41; Secondary 11F30.

Key words and phrases. Hilbert modular forms, Fourier coefficients.

(C)2002 American Mathematical Society 
In this note we extend the Rayleigh quotient method used in [3] to holomorphic Hilbert modular forms. We let $n_{1}=n_{2}=\ldots=n_{p}=1$, and let $K / \mathbb{Q}$ be a totally real number field of degree $p$ with embeddings $\sigma_{1}, \ldots, \sigma_{p}$ into $\mathbb{R}$. Let $\Gamma=\mathrm{SL}_{2}\left(\mathcal{O}_{K}\right)$. Let $f\left(z_{1}, \ldots, z_{p}\right)$ be a Hilbert modular cusp form with respect to $\Gamma$. We say that $f$ has weight $\vec{k}=\left(k_{1}, \ldots, k_{p}\right)$ if, for every $\gamma \in \Gamma$, we have

$$
f\left(\gamma \cdot\left(z_{1}, z_{2}, \ldots, z_{p}\right)\right)=\prod_{i=1}^{p}\left(c^{\sigma_{1}} z_{i}+d^{\sigma_{i}}\right)^{2 k_{i}} f\left(z_{1}, \ldots, z_{p}\right)
$$

where $\gamma=\left(\begin{array}{ll}a & b \\ c & d\end{array}\right)$. If $s_{1}, \ldots, s_{h}$ are the cusps for $\Gamma$ and $U_{j}$ is an element of $\operatorname{SL}_{2}(K)$ that maps the cusp $(i \infty, \ldots, i \infty)$ to $s_{j}$, then $f$ admits a Fourier expansion of the form

$$
f\left(z_{1}, \ldots, z_{p}\right) \mid U_{j}=\sum_{\overrightarrow{0} \neq \vec{l} \in \Lambda_{j}^{*}} a_{\vec{l}}^{(j)} \exp (2 \pi i(\vec{l} \cdot \vec{z}))
$$

where $\vec{z}=\left(z_{1}, \ldots, z_{p}\right), z_{m}=x_{m}+i y_{m}, \Lambda_{j}^{*}$ is the dual of $\Lambda_{j}$, which is a translation lattice in $\mathcal{O}_{K}$ fixing $s_{j}$; see [1, pp. 44-45] or [9]. It is known (see [1, p. 51]) that $a_{\vec{l}}^{(j)}=0$ if $\vec{l} \notin\left(\mathbb{R}^{+}\right)^{p}$.

For $\eta$ an ideal in $\mathcal{O}_{K}$, let $\Gamma(\eta)$ be the principal congruence subgroup of $\Gamma$ of level $\eta$, i.e., $\Gamma(\eta)=\operatorname{ker}\left(\mathrm{SL}_{2}\left(\mathcal{O}_{K}\right) \longrightarrow \mathrm{SL}_{2}\left(\mathcal{O}_{K} / \eta\right)\right)$. Then $\Gamma(\eta)$ is a normal subgroup of $\Gamma$. Let $t_{1}, \ldots, t_{h(\eta)}$ be the cusps for $\Gamma(\eta)$, and let $U_{i} \in \operatorname{SL}_{2}(K) \operatorname{map}(i \infty, \ldots, i \infty)$ to $t_{i}$. As before, if $f\left(z_{1}, \ldots, z_{p}\right)$ is a Hilbert modular cusp form with respect to $\Gamma(\eta)$ of weight $\vec{k}$, then $f$ has a Fourier expansion at the cusp $t_{i}$ of the form

$$
f\left(z_{1}, \ldots, z_{p}\right) \mid U_{i}=\sum_{\overrightarrow{0} \neq \vec{l} \in \Omega_{i}^{*}} c_{\vec{l}}^{(i)} \exp (2 \pi i(\vec{l} \cdot \vec{z}))
$$

where $\Omega_{i}^{*}$ is the dual lattice in $K$ to the translation lattice in $\eta$ that fixes $t_{i}$.

Our first theorem is the following:

Theorem 1. Let $f\left(z_{1}, \ldots, z_{p}\right)$ be a holomorphic Hilbert modular cusp form for $\Gamma$ of weights $\left(k_{1}, \ldots, k_{p}\right)$ with Fourier expansion at the $j$-th cusp given by (1.1). There is a constant $C_{K}$ so that if $c_{\vec{l}}^{(j)}=0$ for every $j$ and for every $\vec{l} \in \Lambda_{j}^{*}$ with $|\vec{l}| \leq C_{K}\left(\sum_{i=1}^{p} k_{i}\right)$, then $f \equiv 0$.

In the rest of this article the constant in $O(H(\vec{k}))$ depends on the group $\Gamma$ (but not on $\vec{k}$ ) and the constant in $O(H(N))$ depends on the group $\Gamma$ and $\vec{k}$ (but not on $N)$.

Corollary 2. The dimension of the space of Hilbert modular forms of weight $\vec{k}$ for $\Gamma$ is bounded by $O\left(|\vec{k}|^{p}\right)$.

Corollary 2 was originally proved in [8] for $k_{1}=k_{2}=\cdots=k_{p}$ using the maximum principle and the norm $\|z\|=\max \left|z_{i}\right|$ in $\mathbb{C}^{p}$. The importance of Corollary 2 is that it implies that the set of automorphic functions forms a field of algebraic functions in $p$ independent variables.

Our proof avoids the maximum principle and norm and follows the modern point of view that holomorphic cusp forms (as well as nonholomorphic cusp forms) are eigenfunctions of certain self-adjoint operators, which are restrictions of the Casimir operator of the Lie algebra $\operatorname{sl}_{2}\left(\mathcal{O}_{K}\right)$ to subspaces of $L^{2}\left(\Gamma \backslash \mathrm{SL}_{2}\left(\mathcal{O}_{K}\right)\right)$ of various weights. The Rayleigh quotient produces for such eigenfunctions their eigenvalues. 
It should be noted that one can show the existence of a polynomial bound of some degree on the dimension of the space of Hilbert modular forms using the Hilbert polynomial for the cohomology of Hilbert modular varieties; see [1, p. 116].

The main theorem in this article is the following.

Theorem 3. Let $f$ be a holomorphic Hilbert modular form for $\Gamma(\eta)$ of weight $\vec{k}=$ $\left(k_{1}, \ldots, k_{p}\right)$, where $\eta$ has norm $N$, and suppose that $S L_{2}\left(\mathcal{O}_{K}\right)$ has only one cusp. Let $f$ have a Fourier expansion at $t_{j}$ as in (1.2). There is a constant $C_{K, \vec{k}}$ so that if $c_{\vec{l}}^{(j)}=0$ for every $j$ and for every $\vec{l} \in \Omega_{j}^{*}$ with $|\vec{l}| \leq C_{K, \vec{k}} N$, then $f \equiv 0$.

Corollary 4. The dimension of the space of Hilbert modular forms of weight $\vec{k}$ for $\Gamma(\eta)$ is bounded by $O\left(N^{p+4}\right)$.

For the case $p=1$ our method is not sharp, because it requires that we check the Fourier coefficients at all cusps. See Remark 6 for an improvement.

Remark 5. We work with the principal congruence subgroup $\Gamma(\eta)$ and we assume that $\Gamma$ has only one cusp for simplicity of the exposition only. One can also use the same arguments for Hilbert modular forms of half-integral weights, because the multiplier systems have absolute value 1 .

\section{Proof of the theorems}

2.1. Fundamental domains. Let $\mathcal{D}(1)$ be the fundamental domain for the action of $\Gamma$ on $\mathbb{H} \times \cdots \times \mathbb{H}$. Let $s_{1}, \ldots, s_{h}$ be the cusps for $\Gamma$. There is a decomposition $\mathcal{D}(1)=\bigcup_{j=1}^{h} \mathcal{D}_{j}$, where each $\mathcal{D}_{j}$ contains a single cusp $s_{j}$. Let $\Gamma_{\infty}^{j}$ be the stabiliser of $s_{j}$ in $\Gamma$ and $\Gamma_{j}=U_{j}^{-1} \Gamma_{\infty}^{j} U_{j}$. From [1, p. 69] it follows that there are Siegel sets $S_{j}$ of the form

$$
U_{j}^{-1} S_{j}=\left\{\left(z_{1}, \ldots, z_{p}\right) \in\left(\Gamma_{j} \backslash \mathbb{H} \times \cdots \times \mathbb{H}\right), \quad y_{n} \geq \Theta, \quad n=1,2, \ldots, p\right\}
$$

for $1 \leq j \leq h$ and for some positive real number $\Theta$ with the property that $\mathcal{D}_{j} \subset S_{j}$ and each $S_{j}$ is contained in the union of a finite number of copies of $\mathcal{D}(1)$.

For Theorem 3 we assume that $\mathrm{SL}_{2}\left(\mathcal{O}_{K}\right)$ has only one cusp $s_{1}$ and we denote by $S_{1}$ the Siegel set for $\Gamma$. Let $\mathcal{D}(\eta)$ be the fundamental domain of $\Gamma(\eta)$. Then $\mathcal{D}(\eta)$ has a decomposition as $\mathcal{D}(\eta)=\bigcup_{j=1}^{h(\eta)} \mathcal{D}_{j}(\eta)$.

We construct Siegel sets $S_{j}(\eta)$ covering $\mathcal{D}(\eta)$ as follows. Choose $U_{j}$ in $\mathrm{SL}_{2}\left(\mathcal{O}_{K}\right)$. Let $\Gamma_{\infty}^{j}(\eta)$ be the stabilizer in $\Gamma(\eta)$ of $t_{j}$, and $\Gamma_{j}(\eta)=U_{j}^{-1} \Gamma_{\infty}^{j}(\eta) U_{j}$. The Siegel sets are defined as

$$
U_{j}^{-1} S_{j}(\eta)=\bigcup_{m=1}^{k(\eta)} \gamma_{m} S_{1},
$$

where $\gamma_{1}, \gamma_{2}, \ldots, \gamma_{k(\eta)}$ are coset representatives of $\Gamma_{j}(\eta)$ in $\Gamma_{1}$. Because $\Gamma(\eta)$ is normal in $\Gamma$, the number of coset representatives $k(\eta)$ is the same for all cusps of $\Gamma(\eta)$ and we set $M=k(\eta)$. From (2.1) it follows that $\Theta$ can be taken to be the same for all $\eta$. The properties of the $S_{j}(\eta)$ that we need are: $\mathcal{D}_{j}(\eta) \subset S_{j}(\eta)$ and $S_{j}(\eta)$ is contained in a number of copies of $\mathcal{D}(\eta)$ equal to the number of copies of $\mathcal{D}(1)$ covering $S_{1}$. Consequently this number is independent of $\eta$. This follows from the rules of joining copies of $\mathcal{D}(1)$ to obtain $\mathcal{D}(\eta)$. 
As in [10, p. 22], the number of cusps $h(\eta)$ for the groups $\Gamma(\eta)$ is given by the formula

$$
h(\eta)=[\Gamma: \Gamma(\eta)] / M .
$$

Since $\left[\mathcal{O}_{K}: \eta\right]=N,[\Gamma: \Gamma(\eta)] \leq N^{3}$. Since $\Gamma_{1}=\left\{\left(\begin{array}{cc}\epsilon & x \\ 0 & \epsilon^{-1}\end{array}\right)\right\}$ with $\epsilon \in \mathcal{O}_{K}^{*}, x \in \mathcal{O}_{K}$, we see that $N^{2} \geq M \geq N$. It follows that $h(\eta) \leq N^{2}$ and $\left[\Omega_{j}^{*}: \Lambda_{1}^{*}\right] \leq N^{2}$.

\subsection{Action of the Laplace operators. Let}

$$
F\left(z_{1}, \ldots, z_{p}\right)=y_{1}^{k_{1}} \cdots y_{p}^{k_{p}} f\left(z_{1}, \ldots, z_{p}\right) .
$$

If $\Delta_{k, i}$ denotes the $k_{i}$ Laplace operator

$$
\Delta_{k, i}=y_{i}^{2}\left(\frac{\partial^{2}}{\partial x_{i}^{2}}+\frac{\partial^{2}}{\partial y_{i}^{2}}\right)-2 i k_{i} y_{i} \frac{\partial}{\partial x_{i}}
$$

which acts on the $i$-th variable of $F\left(z_{1}, \ldots, z_{p}\right)$, then $F$ satisfies

$$
\Delta_{k, i} F=k_{i}\left(k_{i}-1\right) F \text {. }
$$

Let $K_{k_{i}}=\left(z_{i}-\bar{z}_{i}\right) \frac{\partial}{\partial z_{i}}+k_{i}$ and $L_{k_{i}}=\left(\bar{z}_{i}-z_{i}\right) \frac{\partial}{\partial \bar{z}_{i}}-k_{i}$. Then,

$$
\Delta_{k, i}=L_{k_{i}+1} K_{k_{i}}+k_{i}\left(1+k_{i}\right)
$$

see [7. From equation (9.1) of [7], we know that for any domain $X \in \mathbb{H}$ and functions $f, g$ on $\mathbb{H}$, we have

$$
-\int_{\partial X} \bar{g} K_{k_{i}} f \frac{d z}{y}=\int_{X}\left(\bar{g} L_{k_{i}+1} K_{k_{i}} f+\overline{K_{k_{i}} g} K_{k_{i}} f\right) \frac{d x d y}{y^{2}} .
$$

Letting $f=g=F$, and $X=\mathcal{D} \cap \mathbb{H}_{(i)}$ (where $\mathbb{H}_{(i)}$ denotes the $i$-th copy of $\mathbb{H}$ in $\mathbb{H} \times \cdots \times \mathbb{H})$ in $(2.3)$, we have

$$
\begin{aligned}
\int_{\partial X} \bar{F} K_{k_{i}} F \frac{d z_{i}}{y_{i}} & =-\int_{X}\left(\bar{F}\left(\Delta_{k, i}-k_{i}\left(k_{i}+1\right)\right) F+\overline{K_{k_{i}} F} K_{k_{i}} F\right) \frac{d x_{i} d y_{i}}{y_{i}^{2}} \\
& =-\int_{X}\left(\left(k_{i}\left(k_{i}-1\right)-k_{i}\left(k_{i}+1\right)\right)|F|^{2}+\left|K_{k_{i}} F\right|^{2}\right) \frac{d x_{i} d y_{i}}{y_{i}^{2}} .
\end{aligned}
$$

Integrating over all the $z_{j}$ 's, $j \neq i$, we have

$$
-\int_{\partial \mathcal{D}} \bar{F} K_{k_{i}} F d S=\int_{\mathcal{D}}\left(\left(k_{i}\left(k_{i}-1\right)-k_{i}\left(k_{i}+1\right)\right)|F|^{2}+\left|K_{k_{i}} F\right|^{2}\right) d V,
$$

where $d S$ and $d V$ denote the surface and volume measure on the fundamental domain $\mathcal{D}$ of the group under investigation. We denote $h^{\prime}$ the number of cusps. By the construction of the fundamental domain, we see that the left-hand side of equation (2.5) is zero, because $\bar{F} K_{k_{i}} F d S$ is invariant under the action of $\Gamma$. Naturally all this amounts to the fact that $\Delta_{k, i}-k_{i}\left(k_{i}+1\right)$ is self-adjoint and corresponds to the quadratic form $\left\|K_{k_{i}} F\right\|_{2}^{2}$. Using (2.5) , we have for every $i$

$$
\int_{\mathcal{D}}\left|K_{k_{i}} F\right|^{2} d V=\int_{\mathcal{D}}\left(2 k_{i}\right)|F|^{2} d V
$$

and, hence,

$$
\sum_{i=1}^{p} \int_{\mathcal{D}}\left|K_{k_{i}} F\right|^{2} d V=\sum_{i=1}^{p} \int_{\mathcal{D}}\left(2 k_{i}\right)|F|^{2} d V
$$


Using the properties of the Siegel sets $S_{j}$ covering $\mathcal{D}$, we have

$$
\frac{\sum_{i} \int_{S_{j}}\left|K_{k_{i}} F\right|^{2} d V}{\int_{S_{j}}|F|^{2} d V} \leq C \frac{\sum_{i} \iint_{\mathcal{D}}\left|K_{k_{i}} F\right|^{2} d V}{\iint_{\mathcal{D}_{j}}|F|^{2} d V}
$$

for some constant $C$. Taking the minimum of the left-hand side over all $j$, and adding over all cusps, we get

$$
\sum_{k=1}^{h^{\prime}} \int_{\mathcal{D}_{k}}|F|^{2} d V \min \left(\frac{\sum_{i} \int_{S_{j}}\left|K_{k_{i}} F\right|^{2} d V}{\int_{S_{j}}|F|^{2} d V}\right) \leq C h^{\prime} \sum_{i} \int_{\mathcal{D}}\left|K_{k_{i}} F\right|^{2} d V .
$$

We deduce, using (2.6) and (2.9), that

$$
\min \left(\frac{\sum_{i} \int_{S_{j}}\left|K_{k_{i}} F\right|^{2} d V}{\int_{S_{j}}|F|^{2} d V}\right) \leq C h^{\prime} \sum_{i=1}^{p}\left(2 k_{i}\right) .
$$

Proof of Theorem [1. We let $\mathcal{D}=\mathcal{D}(1)$ and $h^{\prime}=h$. Substituting the Fourier expansion of $f$ from (1.1) at the cusp at which the minimum is attained and using Parseval's formula, we get

$$
\sum_{i=1}^{p} \frac{\int \sum_{\vec{l} \in \Lambda_{j}^{*}}\left|a_{\vec{l}}^{(j)}\right|^{2}\left|k_{i}-2 \pi l_{i} y_{i}\right|^{2} \prod_{i=1}^{p}\left(y_{i}^{2 k_{i}} e^{-4 \pi l_{i} y_{i}} d y_{i} / y_{i}^{2}\right)}{\int \sum_{\vec{l} \in \Lambda_{j}^{*}}\left|a_{\vec{l}}^{(j)}\right|^{2} \prod_{i=1}^{p}\left(y_{i}^{2 k_{i}} e^{-4 \pi l_{i} y_{i}} d y_{i} / y_{i}^{2}\right)} \leq 2 C h \sum_{i=1}^{p} k_{i}
$$

where the integrals are over the Siegel set $S_{j}$. Each of the $y_{i}$ 's is bounded below by $\Theta$. If $a_{\vec{l}}^{(j)}=0$ for $|\vec{l}| \leq C(\vec{k})$, then at least one of the $l_{i}$ would be large relative to $k_{i}$. Using (2.11), we have

$$
\left|k_{i}-2 \pi l_{i} \Theta\right|^{2} \leq 2 C h \sum_{i=1}^{p} k_{i},
$$

where $|\vec{l}| \geq C(\vec{k})$. If $C(\vec{k})$ is chosen as $C_{K} \sum_{i} k_{i}$, where $C_{K}$ is some appropriately chosen constant depending only on $K$, then the left-hand side of equation (2.12), being quadratic in $k_{i}$, can be made larger than the right-hand side which is linear in $k_{i}$, contradicting (2.12). We conclude that $F$ and hence $f$ must be identically zero.

Proof of Corollary 2 A ball of radius $R$ in $\mathbb{R}^{p}$ contains $O\left(R^{p}\right)$ vectors in $\Lambda^{*}$, and we let $R=O\left(\sum_{i} k_{i}\right)$.

Proof of Theorem . We let $\mathcal{D}=\mathcal{D}(\eta)$ and $h^{\prime}=h(\eta)$. Using the Fourier expansion from (1.2) in equation (2.10) at the cusp at which the minimum is attained, and using the notation of subsection 2.1, we have

$$
\sum_{i=1}^{p} \frac{\int \sum_{\vec{l} \in \Omega_{j}^{*}}\left|c_{\vec{l}}^{(j)}\right|^{2}\left|k_{i}-2 \pi l_{i} y_{i}\right|^{2} \prod_{i=1}^{p}\left(y_{i}^{2 k_{i}} e^{-4 \pi l_{i} y_{i}} d y_{i} / y_{i}^{2}\right)}{\int \sum_{\vec{l} \in \Omega_{j}^{*}}\left|c_{\vec{l}}^{(j)}\right|^{2} \prod_{i=1}^{p}\left(y_{i}^{2 k_{i}} e^{-4 \pi l_{i} y_{i}} d y_{i} / y_{i}^{2}\right)} \leq 2 C h(\eta) \sum_{i=1}^{p} k_{i} .
$$

As in the proof of Theorem [1] if $c_{\vec{l}}^{(j)}=0$ for $|\vec{l}| \leq C(N, \vec{k})$, we have

$$
\left|k_{i}-2 \pi l_{i} \Theta\right|^{2} \leq 2 C h(\eta) \sum_{i=1}^{p} k_{i} .
$$


If $C(N, \vec{k})$ is chosen as $C_{K, \vec{k}} h(\eta)^{1 / 2}$, then the left-hand side of (2.14) grows faster than the right-hand side as a function of $N$ which is a contradiction. Thus $F$ would have to be identically 0 .

To conclude Theorem 3, we see from subsection 2.1 that $h(\eta) \leq N^{2}$.

Proof of Corollary 4 The index of $\Lambda_{1}^{*}$ in $\Omega_{j}^{*}$ is at most $N^{2}$ and consequently the number of lattice points of $\Omega_{j}^{*}$ with length $\leq R$ is $O\left(N^{2} R^{p}\right)$. We take $R=O(N)$, by Theorem 3 and there exist at most $N^{2}$ cusps.

Remark 6. When $p=1$ Corollary 4 gives a bound $O\left(N^{5}\right)$. However, it can be strengthened by noticing that $M=N$, and, hence, the dimension is $O\left(N^{4}\right)$. Actually [10,1.6.4, p. 23] gives the genus of $\Gamma(N) \backslash \mathbb{H}$ and the correct order of growth of the space of cusp forms of fixed weight is $O\left(N^{3}\right)$. The discrepancy is the result of our need to check the Fourier coefficients at all cusps.

\section{REFERENCES}

[1] Freitag, E.: Hilbert Modular Forms. Springer Verlag, New York, Berlin, Heidelberg, 1990. MR 91c:11025

[2] Goldfeld, D.; Hoffstein, J.: On the number of Fourier coefficients that determine a modular form. A tribute to Emil Grosswald: number theory and related analysis, 385-393, Contemp. Math., 143, Amer. Math. Soc., Providence, RI, 1993. MR 94b:11037

[3] Huntley, J.: Spectral multiplicity on products of hyperbolic spaces. Proc. Amer. Math. Soc. 111 (1991), no. 1, 1-12. MR 91d:11055

[4] Jacquet, H.; Piatetskii-Shapiro, I. I.; Shalika, J. A.: Rankin-Selberg convolutions. Amer. J. Math. 105 (1983), no. 2, 367-464. MR 85g:11044

[5] Moreno, C. J.: Analytic proof of the strong multiplicity one theorem. Amer. J. Math. 107 (1985), no. 1, 163-206. MR 86m:22027

[6] Murty, M. R.: Congruences between modular forms. Analytic number theory (Kyoto, 1996), 309-320, London Math. Soc. Lecture Note Ser., 247, Cambridge Univ. Press, Cambridge, 1997. MR 2000c:11073

[7] Roelcke, W.: Das Eigenwertproblem der automorphen Formen in der hyperbolischen Ebene. I, II. Math. Ann. 167 (1966), 292-337; ibid. 168 (1966) 261-324. MR 39:4386

[8] Piatetskii-Shapiro, I. I.: Estimate of the dimensionality of the space of automorphous forms for certain types of discrete groups. (Russian) Dokl. Akad. Nauk SSSR (N.S.) 113 (1957) 980-983.

[9] Shimizu, H.: On discontinuous groups operating on the product of the upper half planes. Ann. of Math. (2) $\mathbf{7 7}$ (1963) 33-71. MR 26:2641

[10] Shimura, G.: Introduction to the arithmetic theory of automorphic function, Princeton University Press, Princeton, N.J., 1971. MR 47:3318

Department of Mathematics and Statistics, McGill University, Montreal, Quebec, CANADA H3A 2K6

E-mail address: sbaba@math.mcgill.ca

School of Mathematics, Harish-Chandra Research Institute, Chhatnag Road, Jhunsi, Allahabad, 211019, India

E-mail address: kalyan@mri.ernet.in

Department of Mathematics and Statistics, McGill University, Montreal, Quebec, Canada H3A 2K6

E-mail address: petridis@math.mcgill.ca 